\title{
Game-based learning: juego de cartas para aprender Ciencia
}

\section{Carla Álvarez Romero ${ }^{a}$ y Laura Osete Cortina ${ }^{b}$}

anstituto Universitario de Restauración del Patrimonio. Universitat Politècnica de València, Camino de Vera s/n, 46022, València. e-mail: carla.alvarez.romero@gmail.com b'Instituto Universitario de Restauración del Patrimonio. Universitat Politècnica de València, Camino de Vera s/n, 46022, València. E-mail: losete@crbc.upv.es.

\begin{abstract}
The use of games in the classroom allows the learning of formal concepts in a favourable climate where the students are incentivized for the achievement of a challenge increasing the motivation and the development of competences, thereby promoting the cognitive processes. Taking advance of the features that games provides for learning, an activity aimed at the resolution of specific problems in the field of Conservation and Restoration by the determination of instrumentation and examination methods for the documentation of Cultural Goods is designed.
\end{abstract}

Keywords: Game based learning, motivation, PBL, restoration, instrumental techniques, works of art, cooperative learning.

\footnotetext{
Resumen

La incorporación de elementos lúdicos en el aula permite el aprendizaje de conceptos formales en un entorno favorable en el que los alumnos son incentivados en la consecución de desafios potenciando la motivación y el desarrollo de competencias favoreciendo de esta manera el proceso cognitivo. Aprovechando las características que proporciona los juegos al aprendizaje, se diseña una actividad orientada a la resolución de problemáticas específicas en el ámbito de la Conservación y Restauración mediante la determinación de los instrumentos y métodos de examen necesarios para la documentación de los Bienes Culturales.
}

Palabras clave: aprendizaje basado en juegos, motivación, aprendizaje basado en problemas, restauración, técnicas instrumentales, obras de arte, aprendizaje cooperativo.

\section{1.-Introducción}

Teniendo en cuenta el modelo de formación por competencias dentro de los planes de estudio del máster en Conservación y Restauración de Bienes Culturales de la Universitat Politècnica de València (UPV), y ante la necesidad de adquirir conocimientos multidisciplinares y desarrollar habilidades cognitivas para la toma de decisiones técnicas, se encuentra la 
asignatura "Técnicas Instrumentales para el desarrollo de la experimentación en la Conservación y Restauración del Patrimonio". Se trata de una asignatura optativa que se imparte en el segundo curso del Máster en Conservación y Restauración de Bienes Culturales de la Facultad de Bellas Artes de la UPV. El número de alumnos matriculados durante el curso académico 2018-2019 fue de 14, que supone el 50\% de los alumnos matriculados en el Máster.

Para la adquisición de la competencia transversal "Instrumental específica" (CT-13 de la UPV), consistente en la capacidad de identificar las herramientas más adecuadas en cada caso, conociendo sus utilidades y siendo capaz de integrarlas y combinarlas para poder resolver un problema, realizar un proyecto o un experimento (UPV, 2019), se parten de los siguientes resultados de aprendizaje:

- Aplicar las técnicas instrumentales de análisis para la resolución de una problemática en Conservación y Restauración.

- Decidir y justificar el/los ensayos físico-mecánicos que se emplearían para la resolución de una problemática en Conservación y Restauración.

Esta asignatura, de marcado carácter científico, constituye un reto tanto para los docentes como para los alumnos que, debido a la gran especialización de los perfiles académicos, en la mayoría de los casos, carecen de una base científica sólida que permita un aprendizaje significativo de estos conocimientos técnicos. Por todo ello, los contenidos de esta asignatura y las metodologías de enseñanza-aprendizaje aplicadas, deben adaptarse a las características particulares de los estudiantes procedentes del ámbito humanístico (Bellas Artes) con el fin de facilitar el aprendizaje.

La motivación es uno de los factores más influyentes en los procesos cognitivos (Vélez Osorio, 2016; Contreras Espinosa, 2016; Cabezas y Alonso Granado, 2015) y la adquisición de nuevos conocimientos y destrezas está íntimamente relacionada con el papel activo del alumno y su predisposición frente al proceso de aprendizaje (Fernández Solo de Zaldívar, 2015; Kim, 2015). Asimismo, el aprendizaje basado en juegos (game-based learning) se trata de una metodología en la que el escenario lúdico toma protagonismo contribuyendo a la consecución de competencias transversales y específicas necesarias para el futuro desempeño profesional (Villalustre Martínez y del Moral Pérez, 2015; Rodríguez Muñoz, 2015). Por todo ello, se ha diseñado una actividad basada en juegos mediante la que se busca generar un buen clima en el aula, donde los alumnos se impliquen en el trabajo en equipo (competencia transversal CT-06 de la UPV) favoreciendo la participación y el aprendizaje de todos los participantes. 


\section{2.- Objetivos}

Mediante la implementación de estrategias de gamificación se pretende favorecer la implicación de los estudiantes mediante la motivación por el aprendizaje y el desarrollo de habilidades cognitivas para la toma de decisiones. Se plantea una actividad realizada en el aula con la finalidad de:

- Generar el pensamiento práctico para la resolución de problemas reales en el contexto de su perfil profesional mediante la aplicación de instrumentación técnica específica

- Incrementar la motivación a partir de la propuesta de experiencias de juego en contextos formativos aplicando metodologías de aprendizaje basado en problemas

- Desarrollar habilidades sociales y comunicativas mediante el aprendizaje cooperativo

- Lograr una mayor implicación de los estudiantes para favorecer el aprendizaje activo y colaborativo

\section{3.- Desarrollo de la innovación}

Para la consecución de estos resultados de aprendizaje, se diseñó la estructura de un juego mediante el cual se trabajarán en el aula problemáticas específicas que los alumnos, como futuros restauradores, podrían encontrarse en su ámbito profesional. Esta profesión, claramente interdisciplinar, requiere de la integración de conocimiento, atravesando las barreras propias del conocimiento fragmentado en disciplinas y materias. Por todo ello, se pretendía que esta actividad reuniera las características que se fomentan en un escenario lúdico en el que prima la implicación del alumno y la motivación, sumado a las características que proporcionan las metodologías de aprendizaje basadas en problemas como proceso de indagación para la resolución de procesos complejos. La suma de estas características pretende que el estudiante sea el protagonista de su propio proceso de aprendizaje, y que con ello sea capaz de desarrollar las habilidades y actitudes necesarias para su futuro profesional.

El juego consiste en el planteamiento previo de un problema real en el que se muestra a los alumnos la fotografía de un objeto artístico o arqueológico que requiere de un proceso de intervención con el fin de asegurar su conservación/restauración. En la Fig. 1 se muestran dos de los ejemplos planteados. Para ello, se deben realizar propuestas para la aplicación de técnicas de examen físico y químico que proporcionen información para evaluar el estado de deterioro del Bien Cultural y determinar las pautas de intervención adecuadas. 

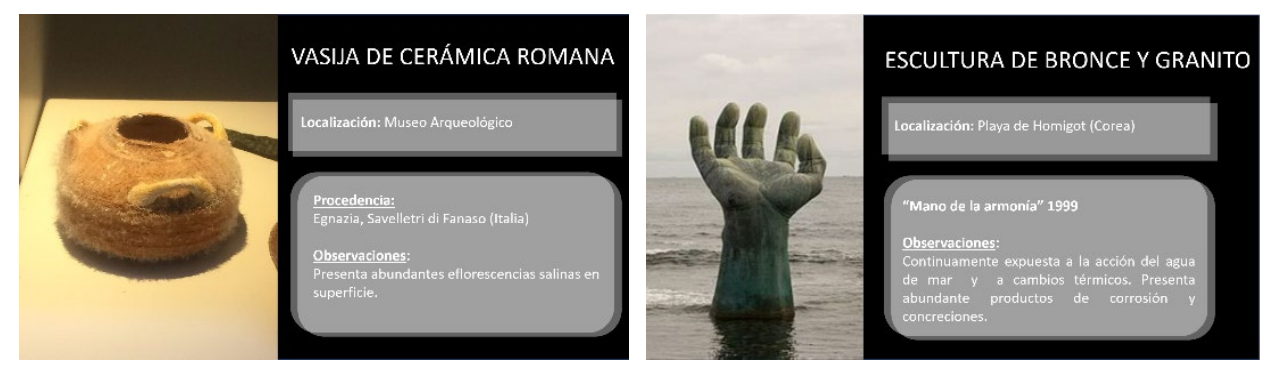

Fig. 1. Ejemplos de problemas planteados

Para el desarrollo del juego se diseñó, expresamente para la actividad, un mazo de 90 cartas, con 11 técnicas de análisis y ensayos físicos habitualmente empleados para el examen científico de Bienes Culturales. En la Fig. 2 se muestra el aspecto de las cartas diseñadas, con la estética propia de las Cartas Magic.

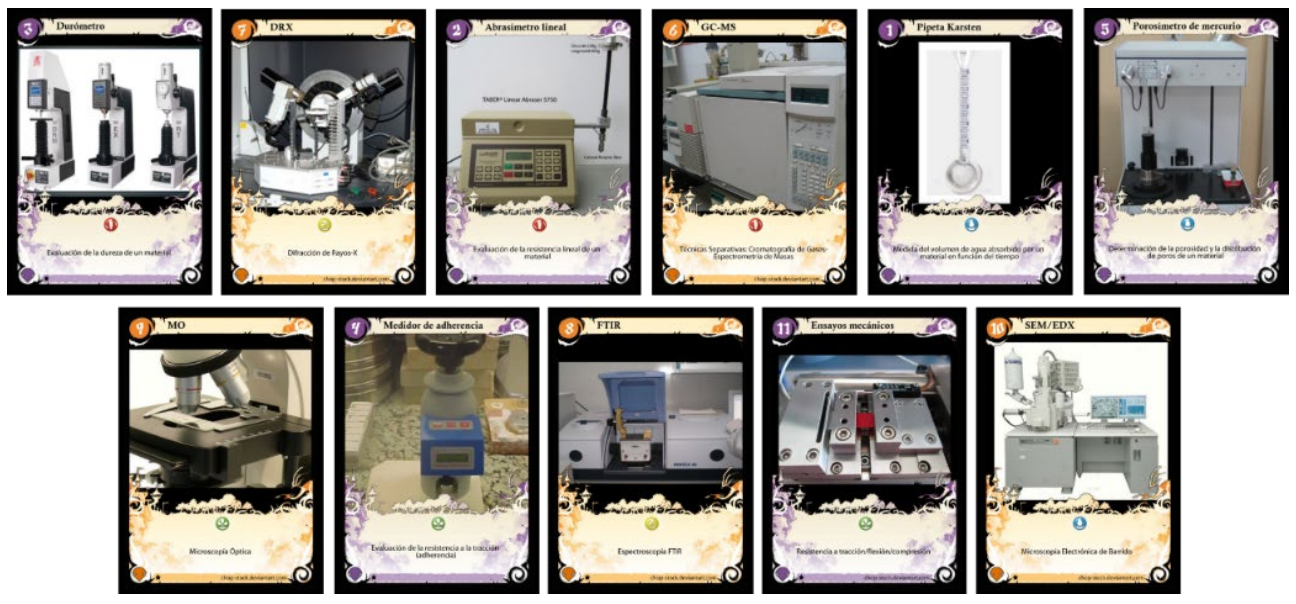

Fig. 2. Cartas diseñadas para el juego

Durante la actividad, los alumnos se dividieron en grupos de cinco participantes y casi la totalidad del mazo de cartas se repartió en los diferentes grupos, de manera que cada uno disponía del mismo numero de cartas, pero con diferentes técnicas y ensayos repetidos.

Una vez planteado el problema, se ponía en funcionamiento un audio con música similar a la de los videojuegos, durante el cual los miembros de cada grupo deliberaban para seleccionar de entre las cartas disponibles aquellas técnicas y ensayos que podrían ser aplicables para el examen científico de esa obra artística u objeto arqueológico planteado en el problema, con el propósito de determinar su estado de conservación y las pautas de intervención.

El primer grupo que terminaba accionaba un pulsador, se detenía la música y se procedía a comprobar si su propuesta de aplicación de análisis y ensayos era adecuada, para lo que debían dar una justificación de la misma. En el caso de que fuera correcta, el grupo se 
descartaba, mientras que, si alguna de las cartas seleccionadas no era adecuada, o la justificación de su aplicación no era correcta, eran penalizados con la pérdida de turno en la siguiente ronda, y además, recibían tantas cartas adicionales como técnicas y/o ensayos inadecuados habían propuesto. El grupo ganador era el que consiguiera quedarse sin cartas primero. En la Fig. 3 se muestra a los alumnos durante el desarrollo del juego.

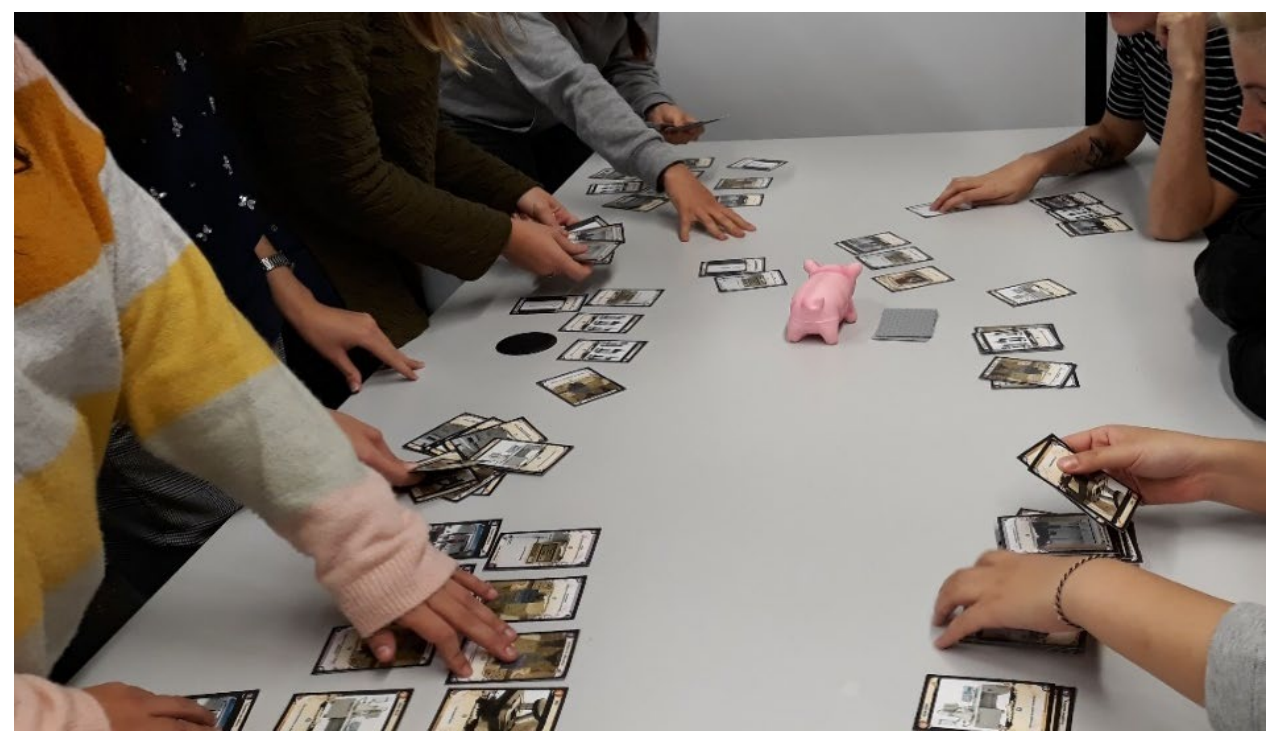

Fig. 3. Detalle de un momento del desarrollo del juego

\section{4.- Resultados}

Con la finalidad de evaluar si mediante la actividad diseñada se había logrado la consecución de los objetivos planteados, se elaboró una rúbrica que permitiera determinar los niveles de realización de los principales objetivos propuestos, centrándonos en los siguientes aspectos:

- La aplicación de las técnicas instrumentales y los ensayos físico-mecánicos en la resolución de problemáticas en Conservación y Restauración (con un peso del 40\%)

- El desarrollo del pensamiento práctico, considerando el análisis adecuado del problema, la realización de propuestas viables y su correcta justificación ( $20 \%$ del peso total)

- El trabajo en equipo, teniendo en cuenta la actitud de trabajo colaborativa con el resto de los miembros del grupo (30\% del peso)

- El grado de la implicación del alumno a partir de su participación activa en el desarrollo del juego ( $10 \%$ del peso total) 
La rúbrica correspondiente se muestra en la siguiente figura (Fig. 4).

\begin{tabular}{|c|c|c|c|c|}
\hline \multicolumn{5}{|c|}{$\begin{array}{l}\text { Valoración del juego } \\
\text { Desarrollo de actitudes y competencias con el juego }\end{array}$} \\
\hline & $\begin{array}{l}\text { D- No alcanzado } \\
\text { 0 puntos }\end{array}$ & $\begin{array}{l}\text { C- En desarrollo } \\
1 \text { puntos }\end{array}$ & $\begin{array}{c}\text { B- Bien } \\
2 \text { puntos Adecuado }\end{array}$ & $\begin{array}{c}\text { A- Excelente } \\
3 \text { puntos Ejemplar }\end{array}$ \\
\hline $\begin{array}{l}\text { Instrumental específica } \\
40 \% \\
\text { Aplicación de las técnicas } \\
\text { instrumentales y ensayos fisico- } \\
\text { mecánicos aplicados a la CyR }\end{array}$ & $\begin{array}{c}\text { D- No alcanzado } \\
\text { No identifica las técnicas y ensayos } \\
\text { avanzados }\end{array}$ & $\begin{array}{l}\text { C- En desarrollo } \\
\text { Identifica las técnicas y los } \\
\text { ensayos avanzados pero no } \\
\text { reconoce su utilidad }\end{array}$ & $\begin{array}{l}\text { B- Bien } \\
\text { Identifica las técnicas y ensayos } \\
\text { avanzados y su función principal }\end{array}$ & $\begin{array}{l}\text { A- Excelente } \\
\text { Identifica funciones adicionales de } \\
\text { las técnicas y ensayos avanzados }\end{array}$ \\
\hline $\begin{array}{l}\text { Desarrollo del pensamiento } \\
\text { práctico } \\
20 \% \\
\text { Analiza el problema, realiza } \\
\text { propuestas viables y las justifica } \\
\text { adecuadamente }\end{array}$ & $\begin{array}{l}\text { D- No alcanzado } \\
\text { No es capaz de realizar propuestas } \\
\text { para la solución del problema }\end{array}$ & $\begin{array}{l}\text { C- En desarrollo } \\
\text { Realiza propuestas incompletas y } \\
\text { no las argumenta }\end{array}$ & $\begin{array}{c}\text { B- Bien } \\
\text { Realiza propuestas incompletas (no } \\
\text { utiliza todos los recursos } \\
\text { disponibles) o no justifica } \\
\text { adecuadamente su elección }\end{array}$ & $\begin{array}{c}\text { A- Excelente } \\
\text { Realiza propuestas válidas, } \\
\text { empleando todos los recursos } \\
\text { disponibles y las justifica } \\
\text { a decuadamente }\end{array}$ \\
\hline $\begin{array}{l}\text { Trabajo en equipo } \\
\mathbf{3 0} \% \\
\text { Presenta una actitud de trabajo } \\
\text { colaborativa aglutinando al resto } \\
\text { de los miembros del equipo }\end{array}$ & $\begin{array}{l}\text { D- No alcanzado } \\
\text { No se integran en el grupo, } \\
\text { presenta una actitud individualista }\end{array}$ & $\begin{array}{c}\text { C- En desarrollo } \\
\text { Se integra parcialmente en el } \\
\text { grupo sin realizar aportaciones } \\
\text { significativas }\end{array}$ & $\begin{array}{c}\text { B- Bien } \\
\text { Realiza un buen trabajo } \\
\text { colaborativo asumiendo un rol en } \\
\text { el equipo para la consecución de } \\
\text { un objetivo común }\end{array}$ & $\begin{array}{c}\text { A- Excelente } \\
\text { Realiza aportaciones significativas } \\
\text { en el equipo, aglutina al resto de } \\
\text { participantes valorando sus } \\
\text { aportaciones }\end{array}$ \\
\hline $\begin{array}{c}\text { Implicación en la actividad } \\
\mathbf{1 0} \% \\
\text { Participación activa de los } \\
\text { estudiantes en el desarrollo de la } \\
\text { actividad }\end{array}$ & $\begin{array}{l}\text { D- No alcanzado } \\
\text { No participa, no realiza } \\
\text { aportaciones al resto del equipo ni } \\
\text { muestra interés por la actividad. }\end{array}$ & $\begin{array}{l}\text { C- En desarrollo } \\
\text { Participa en el juego pero no } \\
\text { realiza aportaciones al grupo. }\end{array}$ & $\begin{array}{l}\text { B- Bien } \\
\text { Se involucra activamente en el } \\
\text { desarrollo del juego. Muestra } \\
\text { interés por la consecución de los } \\
\text { objetivos del juego. }\end{array}$ & $\begin{array}{l}\text { A- Excelente } \\
\text { Contribuye al compromiso colectivo } \\
\text { del equipo, consigue que otros } \\
\text { miembros se impliquen, contagia } \\
\text { su entusiasmo al resto del equipo y } \\
\text { a otros equipos. }\end{array}$ \\
\hline
\end{tabular}

Fig. 4. Rúbrica elaborada para la valoración de los resultados obtenidos a partir de la realización del juego

La rúbrica descrita fue aplicada por las profesoras para la valoración de la actividad desarrollada para los 14 alumnos. Los resultados, en forma de porcentajes obtenidos directamente de la rúbrica, donde el $100 \%$ corresponde al nivel de realización 3 para cada uno de los criterios establecidos, se muestran en la Tabla 1.

Tabla 1. Resultados de la aplicación de la rúbrica

\begin{tabular}{ccccc}
\hline Alumno & $\begin{array}{c}\text { Instrumental } \\
\text { específica }\end{array}$ & $\begin{array}{c}\text { Pensamiento } \\
\text { práctico }\end{array}$ & $\begin{array}{c}\text { Trabajo en } \\
\text { equipo }\end{array}$ & $\begin{array}{c}\text { Implicación en la } \\
\text { actividad }\end{array}$ \\
\hline 1 & 66,7 & 66,7 & 66,7 & 66,6 \\
2 & 66,7 & 66,7 & 66,7 & 66,7 \\
3 & 100 & 100 & 100 & 100 \\
4 & 100 & 100 & 100 & 66,7 \\
5 & 66,7 & 66,7 & 66,7 & 100 \\
6 & 66,7 & 66,7 & 66,7 & 100 \\
7 & 100 & 100 & 100 & 100 \\
8 & 100 & 100 & 100 & 100 \\
9 & 100 & 100 & 100 & 100 \\
10 & 100 & 100 & 100 & 100 \\
11 & 33,3 & 33,3 & 33,3 & 66,7 \\
12 & 66,7 & 66,7 & 66,7 & 100 \\
13 & 66,7 & 66,7 & 66,7 & 100 \\
14 & 100 & 100 & 100 & 100 \\
\hline Valor medio & $80 \pm 20$ & $80 \pm 20$ & $80 \pm 20$ & $90 \pm 20$ \\
y desviación & & & & \\
\hline
\end{tabular}


Se puede observar como, en general, los resultados obtenidos por los 14 alumnos han sido muy satifactorios. Respecto al conocimiento de la correcta aplicación de las técnicas instrumentales y los ensayos fisico-mecánicos aplicados en casos de estudio de conservación y restauración, 7 alumnos han obtenido el máximo valor de la rúbrica, es decir, el 100\% o 3 puntos, siendo capaces de identificar funciones adicionales de las técnicas y los ensayos avanzados explicados en clase, 6 alumnos han obtenido un $66,7 \%$ o 2 puntos, ya que han sido capaces de identificar las funciones principales de dichas técnicas y ensayos, y sólo uno de ellos ha obtenido un valor del 33,3\% o 1 punto debido a que la comprensión del criterio a evaluar aún se encuentra en desarrollo. El valor medio obtenido para el primer criterio evaluable de la rúbrica ha sido de $80 \%$, con una desviación de \pm 20 . Respecto al desarrollo del pensamiento práctico en donde se valora la capacidad de analizar un problema, realizar propuestas viables y justificarlas, los valores obtenidos por los alumnos han sido los siguientes: 7 alumnos han obtenido el $100 \%$ o 3 puntos, 6 alumnos han obtenido un $66,7 \%$ o 2 puntos y 1 alumno ha obtenido un 33,3\% o 1 punto. El valor medio de consecución de este criterio por parte del total del alumnado ha sido del $80 \%$, con una desviación de \pm 20 . Estos mismos resultados han sido obtenidos para el desarrollo del trabajo en equipo, ya que 7 alumnos han obtenido el $100 \%$ de la puntuación, 6 alumnos han sido puntuados con un $66,7 \%$ y un alumno con un $33,3 \%$. El valor medio obtenido ha sido de $80 \%$ con una desviación de \pm 20 . Por último, se valoró la participación y la implicación de forma individual en la actividad. Diez alumnos se implicaron de forma excelente logrando alcanzar el 100\% o 3 puntos de la rúbrica, y 4 de ellos obtuvieron el $66,7 \%$ o 2 puntos. El valor medio obtenido ha sido de $90 \%$ y su desviación de \pm 20 . Como puede advertir, los resultados obtenidos para los cuatro criterios valorables para cada uno de los alumnos se encuentran relacionados entre sí.

De la aplicación de la rúbrica por parte de las profesoras para el grupo de 14 alumnos que constituye la asignatura de Máster en Conservación y Restauración de Bienes Culturales, se extraen los resultados mostrados en la Fig. 5, expresados como valores medios: 


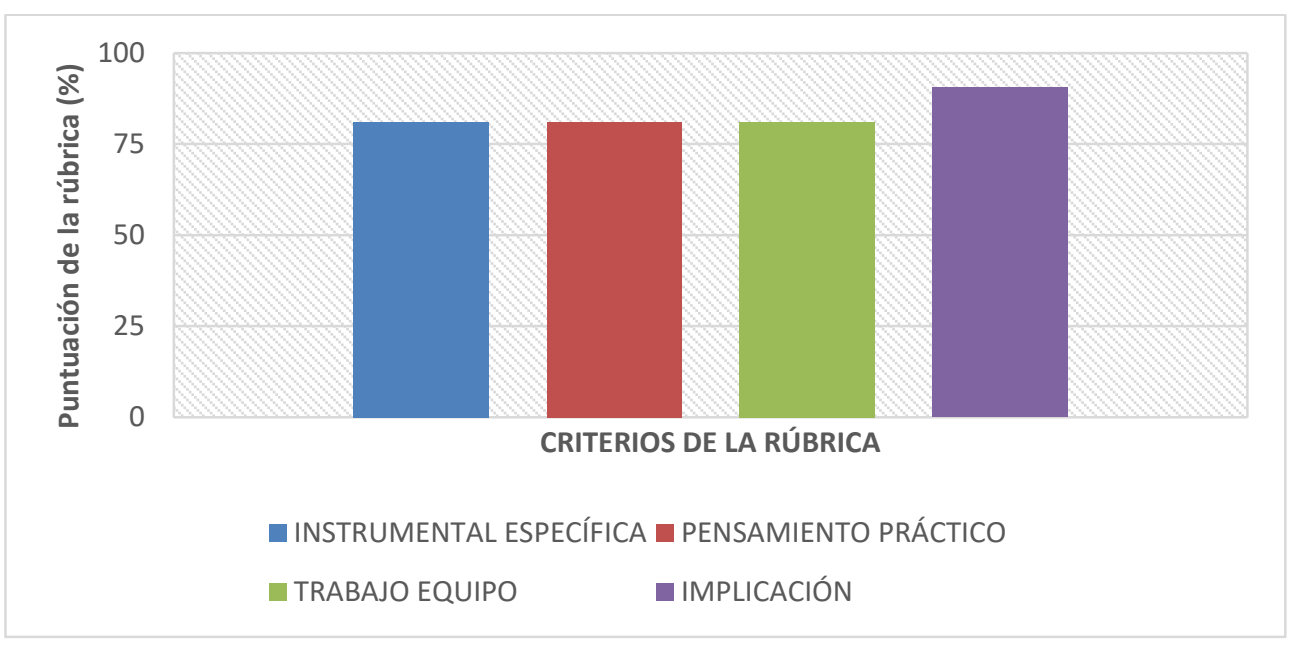

Fig. 5. Resultados medios de las puntuaciones obtenidas de aplicar la rúbrica ( $n=14$ alumnos)

Gracias a los resultados obtenidos, se puede decir que el desarrollo de la actividad ha propiciado un elevado grado de implicación de los alumnos en la participación en la misma.

También es posible asegurar que se ha favorecido en niveles significativos el desarrollo de competencias como el pensamiento práctico y el trabajo colaborativo de los estudiantes, ya que, aunque se trata de una actividad que lleva asociada la competitividad entre grupos, se promueve el trabajo en equipo intergrupo para la consecución de un objetivo común, que es ganar el juego (Wouters et al., 2013).

\section{5.- Conclusiones}

El principal resultado a destacar, es que gracias al desarrollo de la actividad realizada se ha propiciado el incremento del conocimiento del lenguaje científico-técnico, y, concretamente la aplicación de las técnicas analíticas avanzadas y de los ensayos físico-mecánicos a casos de estudio concretos del ámbito de la conservación y la restauración de los bienes culturales, objetivos directamente relacionados con la consecución de la competencia transversal CT-13 de la UPV. La mayoría de los alumnos han sido capaces, a través del juego, de aplicar estos conocimientos y obtener una buena puntuación en la rúbrica diseñada.

La consecución del objetivo del juego, ser el grupo vencedor descartándose de todas las cartas, favorecía la coordinación individual de los alumnos dentro de cada grupo, y su participación, ayudando, además, en el desarrollo de niveles cognitivos complejos que implican el análisis, la resolución de problemas y la justificación o argumentación. El 80\% de los alumnos fueron capaces de analizar los casos planteados y argumentar de manera coherente sus decisiones. 
De manera paralela, se promovió el desarrollo de competencias como el trabajo en equipo y la comunicación efectiva, motivando al alumnado en la experiencia de juego y fomentando la competitividad entre grupos.

A pesar de la simplicidad de la mecánica y dinámica del juego, éste fue recibido muy positivamente por parte de los alumnos, ya que todos ellos se implicaron con la actividad para poder lograr el objetivo final de éste.

\section{6.- Referencias}

CABEZAS, S.G.; ALONSO GRANADO, C. (2015): “Gamificación en entornos educativos universitarios" en Comunicación y pedagogía: Nuevas tecnologías y recursos didácticos, 281-282, pp. 86-91.

CONTRERAS ESPINOSA, R. (2016): "Juegos digitales y gamificación aplicados en el ámbito de la educación" en RIED. Revista Iberoamericana de Educación a Distancia, 19, 2, pp. 27-33.

KIM, B. (2015): Understanding gamification. America: Library Technology Reports.

FERNÁNDEZ SOLO DE ZALDÍVAR, I. (2015): "Juego serio: gamificación y aprendizaje" en Comunicación y pedagogía: Nuevas tecnologías y recursos didácticos, 281-282, pp. 4348.

RODRÍGUEZ MUÑOZ, J. (2015): “Gamificación y diseño” en Comunicación y pedagogía: Nuevas tecnologías y recursos didácticos, 281-282, pp. 55-59.

UNIVERSITAT POLITÈCNICA DE VALÈNCIA. Competencia transversal. Instrumental especifica. $\quad$ http://www.upv.es/contenidos/COMPTRAN/info/955170normalc.html [Consultado: 15 de marzo 2019]

VÉLEZ OSORIO, I.M. (2016): "La gamificación en el aprendizaje de los estudiantes universitarios" en Rastros y Rostros, 18, 22, pp. 27-38.

VILLALUSTRE MARTÍNEZ, L.; DEL MORAL PÉREZ, M.E. (2015): “Gamificación: Estrategia para optimizar el proceso de aprendizaje y la adquisición de competencias en contextos universitarios" en Digital Education Review, 27, pp. 13-31. 
WOUTERS, P.; VAN NIMWEGEN, C.; VAN OOSTENDORP, H.; VAN DER SPECK, E.D. (2013): "A Meta-Analysis of the Cognitive and Motivational Effects of Serious Games" in Journal of Educational Psychology, 4, pp. 1-17. 